\title{
CANCER CACHEXIA IN THORACIC MALIGNANCY: A NARRATIVE REVIEW
}

Andrew C. Kidd ${ }^{1,2}$, Marcin Skrzypski ${ }^{3}$, Mariam Jamal-Hanjani ${ }^{3}$, Kevin G. Blyth ${ }^{1,2}$

${ }^{1}$ Institute of Immunity, Infection and Inflammation, University of Glasgow, United Kingdom

${ }^{2}$ Queen Elizabeth University Hospital, Glasgow, United Kingdom

${ }^{3}$ Cancer Research UK Lung Cancer Centre of Excellence, University College London Cancer Institute, United Kingdom

Corresponding author:

Dr Andrew C. Kidd

Queen Elizabeth University Hospital

1345 Govan Road

Glasgow G51 4TF

Email: andrew.kidd@nhs.net

Telephone: 01414516163

No funding was received for this work.

Word counts:

Abstract: 183 (max. 200 words)

Article: 2,808 (max. 2500 words)

Key words: cancer cachexia, thoracic malignancy, non-small cell lung cancer, malignant pleural mesothelioma 


\begin{abstract}
Purpose of review: Thoracic malignancies are amongst the most lethal of all cancers. Cancer cachexia (CC) lacks unanimously accepted diagnostic criteria and therefore is referenced to as a conceptual framework whereby $\mathrm{CC}$ is "an ongoing loss of skeletal muscle mass (termed sarcopenia), with or without loss of fat mass that cannot be reversed by conventional nutritional support and leads to progressive functional impairment". This review summarises the current evidence base in this field, including imaging techniques currently used to define sarcopenia, inflammatory and metabolic changes associated with the syndrome and ongoing research into potential treatment strategies.
\end{abstract}

Recent findings: Sarcopenia is a key component of the CC syndrome. It is common in patients with both early-stage and advanced NSCLC. Patients with sarcopenia have more treatment-related side effects and poorer overall survival compared with non-sarcopenic patients.

Summary: Early identification of CC may facilitate stratification of patients most-at-risk and initiation of emerging anti-cachexia treatments. If these are proven to be effective, this strategy has the potential to improve tolerance to anti-cancer therapies, improving the quality of life, and perhaps the survival, of patients with thoracic malignancies. 


\section{INTRODUCTION}

Thoracic malignancies are amongst the most lethal of all cancers and include non-small cell lung cancer (NSCLC), small cell lung cancer (SCLC) and malignant pleural mesothelioma (MPM). Previous studies report widely varying estimates of the prevalence of cancer cachexia (CC) in these diseases, with the most data available in NSCLC, but little or no data previously reported in SCLC, MPM and rarer thoracic cancers such as the thymic malignancies. Understanding the significance of $\mathrm{CC}$ in these diseases is complicated by the lack of a universally accepted and applied set of diagnostic criteria. In 2011, an expert international panel defined CC as "a multi-factorial syndrome defined by ongoing loss of skeletal muscle mass (with or without loss of fat mass) that cannot be reversed by conventional nutritional support and leads to progressive functional impairment" ${ }^{\text {" }}$ CC is also associated with various physiological and metabolic events, including systemic inflammation and alterations in basal metabolic rate. In this review, we report the prognostic significance of $\mathrm{CC}$, or its component parts where data regarding $\mathrm{CC}$ per se have not been reported. Since the latter situation is common in this field, we have focused particularly on the constituent elements of CC, including weight loss, sarcopenia (as defined by a variety of imaging tools, which are discussed in some detail) and the systemic inflammatory response. We conclude with an overview of emerging therapeutic strategies that seek to prevent or reverse $\mathrm{CC}$ in patients with thoracic malignancy, with the ultimate aim of improving quality of life and survival by improving tolerance to anti-cancer therapies.

\section{CANCER CACHEXIA SYNDROME}

The prevalence of $\mathrm{CC}$ has been reported to range between $50-80 \%$ in advanced solid cancers $^{2,3}$, with prevalence reported towards the higher end of this range in patients with 
gastrointestinal tract and lung cancers ${ }^{4,5,6}$. The prevalence of CC is less well-defined in terms of specific thoracic malignancy sub-types with most paper simply recording data for 'lung cancer' without differentiation into NSCLC (or its sub-types) or SCLC. No prevalence data exist regarding MPM.

\section{WEIGHT LOSS}

Weight loss is a principal constituent of the CC syndrome and is common in patients with thoracic malignancy ${ }^{7-10}$. Martin et al recently reported a scoring matrix that integrates weight loss with changes in BMI, which could be effectively used to predict survival independently of cancer type, tumour stage, age, sex and performance status in patients with locally advanced or metastatic cancer; $2,561 / 8,160$ (31.4\%) of patients in the training set had respiratory cancer ${ }^{11}$. In NSCLC specifically, weight loss has been associated with adverse outcomes independent of disease stage, performance status and age ${ }^{12}$. Conversely, weight gain in advanced NSCLC patients receiving chemotherapy has been identified previously as an early indicator of clinical benefit ${ }^{13}$. In patients with brain metastases from NSCLC and SCLC, being underweight is an independent prognostic factor ${ }^{14}$. In MPM, previous studies relating to weight loss are discordant, with some studies reporting an adverse association with $\operatorname{prognosis}^{15-18}$ and others not ${ }^{20,21}$.

\section{ANOREXIA}

Anorexia or loss of appetite, is common in patients with $\mathrm{NSCLC}^{28}$ and SCLC $^{29}$. Baseline anorexia has been found to be significantly related to survival in patients with MPM $^{30}$. Anorexia is thought to result from a complex interplay between neuropeptides and cytokines $^{22}$. In animal models, pro-inflammatory cytokines released from tumours induce anorexia by activating neuronal cells expressing anorexigenic pro-opiomelanocortin (POMC) 
in the hypothalamus ${ }^{23}$. POMC and interleukin-1 receptor expression are also suppressed by ghrelin, which increases appetite by up-regulation of agouti-related peptide and neuropeptide $\mathrm{Y}$ expression ${ }^{23}$. Interestingly, patients with stage III and IV NSCLC and anorexia have significantly higher levels of ghrelin compared to patients without anorexia ${ }^{24,25}$, but despite this, patients with $\mathrm{CC}$ do not, in general, report increased appetite. Although researchers have postulated potential reasons for this paradox, mechanisms resulting in increased ghrelin levels in CC remain unclear ${ }^{26}$. Parathyroid hormone-related peptide released by tumours has also been implicated in promoting anorexia and muscle wasting ${ }^{27}$.

\section{ALTERED METABOLISM}

The catabolic effects of $\mathrm{CC}$ result in depletion of skeletal muscle and fat mass ${ }^{31}$. Previous studies have reported that patients with metastatic NSCLC have an elevated resting energy expenditure $(\mathrm{REE})^{32,33}$ which may contribute to the wasting process ${ }^{34,35}$. In these studies, REE was expressed as per kilogram of fat-free mass ${ }^{32}$ and as unadjusted REE (measured by indirect calorimetry) and adjusted REE (measured by dual x-ray absorptiometry (DEXA), potassium-40 measurement and tritiated water dilution ${ }^{33}$. Interesting patients REE have previously been shown to higher again in patients with SCLC compared to those with NSCLC $^{36}$. This observation is concordant with clinical experiences in this classically more aggressive lung cancer sub-type and was associated with higher circulating levels of soluble TNF-receptor 75 and cortisol in the SCLC patients in this study.

\section{SYSTEMIC INFLAMMATION}

The above observation emphasises the important link between elements of the $\mathrm{CC}$ syndrome and the systemic inflammatory response, which can be defined by single measures (commonly the acute phase proteins CRP and albumin), haematological indices (e.g. 
neutrophil: lymphocyte ratio (NLR), platelet: lymphocyte ratio (PLR) or integrated scores, most notably the modified Glasgow Prognostic Score (mGPS; which combines C-Reactive Protein (CRP) and albumin).

In SCLC, measures of a systemic inflammatory response have previously been associated not just with higher REE, but also with adverse outcomes ${ }^{39}$. Hypermetabolic patients with NSCLC who lose weight also have elevated levels of inflammatory mediators, including acute phase proteins ${ }^{37}$. In the same illness, high baseline and progressive increases in NLR have previously been associated with poorer overall survival and weight $\operatorname{loss}^{38}$. Elevated NLR also correlates with sarcopenia in post-operative NSCLC patients ${ }^{40}$, while a useful prognostic index based on BMI and NLR has been reported in patients with advanced NSCLC $^{41}$. An elevated NLR also confers a poor prognosis in male patients with SCLC $^{42}$ and in male and female MPM patients receiving systemic treatment ${ }^{43}$. In MPM, elevated NLR and PLR have also been shown to affect disease-free survival ${ }^{44}$.

Pre-operative increased CRP has previously been associated with a poor prognosis following subsequent resection of NSCLC ${ }^{45}$. Similarly, in MPM pre-treatment CRP levels have previously been associated with subsequent clinical benefit from multi-modality treatments incorporating surgical resection ${ }^{46}$. In advanced NSCLC, low serum albumin has been shown to be a predictive marker of chemotherapy toxicity and adverse survival ${ }^{47}$. Low serum albumin is also a significant prognostic factor in patients with $\mathrm{MPM}^{48}$. In addition, mGPS, which integrates these values, is predictive of survival in patients with both metastatic NSCLC and SCLC ${ }^{49}$.

\section{ALTERED BODY COMPOSITION}


Imaging tools are available for the assessment of body composition including Dual-Energy X-ray Absorptiometry (DEXA), Computed Tomography (CT) and Magnetic Resonance Imaging (MRI). DEXA is used to measure total appendicular lean tissue mass and has commonly been used for the evaluation of cancer cachexia in clinical trials. A recent study that utilised DEXA to define pre-sarcopenia (using a definition of appendicular skeletal muscle mass $\leq 7.26 \mathrm{~kg} / \mathrm{m}^{2}$ for men and $\leq 5.45 \mathrm{~kg} / \mathrm{m}^{2}$ for women) identified this in over half of the patients with MPM studied ${ }^{50}$. However, DEXA is not routinely performed in the clinical setting and measures lean body mass which is not equivalent to muscle mass per se. CT has the advantage of routine availability, facilitating both easier prospective studies and the ability to perform retrospective analyses on existing cohorts. CT images, typically those acquired at the level of the third lumbar vertebrae (L3) can then be used to directly measure muscle and fat indices using readily available software packages and sex-specific cut-off values (see Imaging Considerations for further details).

\section{Sarcopenia}

Sarcopenia is defined as a decrease in skeletal muscle mass. It can be defined using sex- and BMI-specific indices based on measurements of tissue density (Hounsfield Units (HU) derived from a single slice, mid-axial CT image (traditionally at L3) using body composition software tools $\mathrm{s}^{51,52}$. Previous estimates of the prevalence of sarcopenia in thoracic malignancy vary widely. In advanced NSCLC this has been reported to be as high as $60-80 \%{ }^{7}, 50$. However, these studies often do not specify patients' disease stage ${ }^{54-60}$, for example, opting for the terms "unresectable ${ }^{60}$ or 'pre-terminal' ${ }^{\prime 61}$. In earlier stage NSCLC, pre-operative sarcopenia has been reported in $13.9-55.7 \%$ undergoing potentially curative survival resection $^{40,62-66}$. A recent systematic review and meta-analysis identified heterogeneity between study populations (Western versus South East Asian populations), the measurements 
(total skeletal muscle area versus total psoas muscle area) and cut-points (varying sex- or BMI-specific) used to define sarcopenia ${ }^{67}$ as potential reasons for the varying prevalence of sarcopenia reported in NSCLC.

In SCLC and MPM the prevalence of sarcopenia is even less well defined, with no previous data being published in MPM. In SCLC, South Korean researchers recently reported a prevalence of $79.2 \%{ }^{68}$ using locally derived cut-points.

The prognostic importance of sarcopenia has been shown in several previous studies of patients with lung cancer ${ }^{56,69,70}$. In the radically-treatable NSCLC surgical population, preoperative sarcopenia has been associated with poorer outcomes in most studies ${ }^{40,62,64,67}$. A single analysis found no such association but had a short median follow-up period of 26.3 months ${ }^{65}$; (compared to 35.5-61 months in the other cohorts). In patients with early stage lung NSLC who are not fit for surgical resection, stereotactic body radiotherapy (SABR) has recently emerged as a means of delivering radical treatment to inoperable patients (often because of severe respiratory or cardiovascular comorbidities). In this setting, pre-treatment sarcopenia has recently been identified as a risk factor for non-lung cancer death ${ }^{72}$, but interestingly not associated with increased lung cancer-related mortality.

In more advanced NSCLC, patients with sarcopenia prior to initiation of palliative chemotherapy $^{73}$, chemo-radiotherapy ${ }^{77}$ and anti-PD-L1 immunotherapy (with Nivolumab) ${ }^{79}$ have been shown to have increased mortality relative to non-sarcopenic comparators. Sarcopenia has also been associated with reduced tolerance to chemotherapy ${ }^{75,76}$ and has been shown to predict survival regardless of body weight ${ }^{52}$. Patients with NSCLC receiving tyrosine kinase inhibitors have a slower rate of skeletal muscle loss than those receiving cytotoxic chemotherapy. The may reflect lower toxicity from the molecular targeted therapy, 
including less adverse events such as fatigue, loss of appetite and gastrointestinal symptoms that may direct affect sarcopenic change ${ }^{78}$. In patients with SCLC, sarcopenia is also associated with shorter overall survival ${ }^{68,71}$.

\section{Imaging Considerations}

As mentioned earlier, skeletal muscle measurements have traditionally been acquired at the level of L3 across many cancer sub-types. However, this convention poses particular limitations in patients with thoracic malignancy, in whom routine CT acquisitions may not extend this inferiorly. This is especially problematic in retrospective studies, where many patients cannot be included, but also adds complexity and cost to prospective research, since routinely acquired imaging cannot universally be employed. Previous thoracic malignancy researchers have therefore sought alternative measures of skeletal muscle area. Total psoas cross-sectional area, for example, has been associated with prognosis in patients prior to pneumonectomy for locally-advanced NSCLC ${ }^{63}$. Axial CT images acquired at T12 have also been used to study skeletal muscle loss pre- and post-surgery for NSCLC ${ }^{80}$. Low pectoralis muscle area at diagnosis of NSCLC, has also been associated with adverse survival in a previous study ${ }^{81}$. The use of location-specific reference muscle areas has been further extended by recent data which reports that temporal muscle thickness on Head CT imaging is an independent predictor of survival in patients with NSCLC patients and brain metastases ${ }^{83}$. This study quoted a high prior correlation between psoas muscle areas and temporal muscle thickness and more data of this sort are urgently required. Validation studies of skeletal muscle measurements made on thoracic CT images, relative to established L3 values, would be a considerable advance in this field - maximising the size and reducing the cost of future studies. Authors from a single centre in South Korea recently reported high correlation 
between pectoralis muscle area (measured at the aortic arch and at L1) and L3 muscle index in patients with $\operatorname{SCLC}^{82}$, but further studies in this setting and in NSCLC and MPM are needed.

\section{Sarcopenic Obesity}

Sarcopenic obesity describes a clinical phenotype in which obesity $(\mathrm{BMI} \geq 30)$ and low muscle mass occur simultaneously ${ }^{56}$. At present, there have been few studies that have assessed the clinical significance of sarcopenic obesity in patients with thoracic malignancies. In a cohort of Canadian patients, including some with thoracic malignancies, sarcopenic obesity was an independent predictor of adverse survival ${ }^{56}$. In addition, in a recent study of NSCLC patients receiving carboplatin-based doublets, patients with the highest body surface area: lean tissue ratio (a feature associated with sarcopenic obesity) had a higher rate of dose-limiting haematological toxicity compared with patients having the average overall body surface area: lean tissue ratio $^{75}$.

\section{POTENTIAL TREATMENT OF CC IN THORACIC MALIGNANCY}

Better understanding of the biology underlying $\mathrm{CC}$ in thoracic malignancy and development of early detection strategies, as described above, provides an opportunity for therapeutic intervention. The goal of these strategies is to improve QoL, tolerance to cancer therapies and ultimately to improve survival. At present, corticosteroids are the most commonly used CC treatment, having been shown in randomised trials conducted decades ago to improve appetite and QoL ${ }^{87,}{ }^{88}$. However, these drugs are powerful agents with potent immunomodulatory effects and significant toxicities that limit both dose and treatment duration in many patients. They are also contra-indicated, at all but very small doses, in patients receiving immunotherapies, which are becoming increasingly important in the anti- 
cancer armamentarium. Simple nutritional interventions have been shown to increase weight and lean body mass in patients with lung and pancreatic CC receiving chemotherapy ${ }^{85}$. Early, intensive nutritional intervention combined with antiemetics has also been shown to prevent weight loss in patients receiving chemotherapy for $\mathrm{NSCLC}^{86}$.

Increasing interest and understanding of CC biology has recently driven a more targeted approach to $\mathrm{CC}$ drug development, including the evolution of more specific appetite stimulants, anabolic/catabolic transforming agents, anabolic therapies and multi-modal interventions that combine different approaches.

\section{Appetite Stimulation}

Anamorelin is a novel selective ghrelin receptor agonist which has anabolic and appetiteenhancing activities. Anamorelin significantly increased lean body mass but not handgrip strength in the ROMANA $1 \& 2$ trials $^{9}$, which enrolled $\mathrm{x}$ patients with inoperable stage III/IV NSCLC and CC. The ROMANA 3 study found that Anamorelin was well tolerated and safe over $12-24$ weeks $^{90}$ in a further x patients. Anamorelin is not currently approved for use and two further Phase III studies are currently underway.

\section{Anabolic/Catabolic Transforming Agents}

Espindolol which has three pharmacological targets ${ }^{91}$ : blockade of non-selective $\beta$-receptors (thus reducing catabolism), antagonism of central 5-HT1a receptors (potentially reducing fatigue and thermogenesis) and partial agonism of $\beta 2$ receptors (increasing anabolism). The ACT-ONE trial tested two doses of twice daily Espindolol in a randomized, double-blind, 
phase II multicentre trial, which recruited n patients with advanced colorectal or NSCLCrelated CC. In 2016, the trial reported their primary endpoint of a positive weight slope of $0.54 \mathrm{~kg} / 4$ weeks for the high-dose Espindolol group compared with weight loss in the placebo group (negative weight slope of $-0.21 \mathrm{~kg} / 4$ weeks). They also reported improvement in fat free mass and maintenance of fat mass in patients with advanced NSCLC and CC. Espindolol is not currently approved for use but Phase III study is planned.

\section{Anabolic Therapies}

The complex interplay between androgens and androgen receptors can have positive effects on muscle mass and strength by promoting tissue anabolism ${ }^{92}$. Unfortunately, the Prevention and Treatment of Muscle Wasting in Cancer Patients (POWER) 1 and 2 trials, which assessed the efficacy of a non-steroidal selective androgen receptor modulator (Enobosarm) against a placebo in patients with Stage III and IV NSCLC, did not meet the primary endpoints of improvements in total lean body mass or physical function at 3 months ${ }^{93,94}$.

\section{Multimodal Interventions}

A feasibility study of Multimodal Exercise/Nutrition/Anti-inflammatory treatment for Cachexia (Pre-MENAC) was a randomised, phase II study designed to determine the feasibility of a multimodal intervention which included exercise, n-3 polyunsaturated fatty acid nutritional supplements, and an anti-inflammatory agent in patients with advanced lung and pancreatic cancer ${ }^{95}$. The authors recently reported positive feasibility results and a phase III efficacy study is underway; change in body weight at 6 weeks has been selected as the primary end-point ${ }^{95}$. Similarly, the Nutrition and Exercise Treatment for Advanced Cancer (NEXTAC) programme found that early introduction of multimodal interventions was 
associated with high levels of compliance and safety in elderly patients receiving chemotherapy for pancreatic cancer and NSCLC ${ }^{96}$.

\section{CONCLUSION}

CC remains an understudied complication of the thoracic malignancies, including NSCLC, SCLC and MPM. Estimates regarding the prevalence of CC in NSCLC vary greatly and little data exist in SCLC or MPM. From the data that have been reported, there is general agreement that $\mathrm{CC}$ is associated with reduced tolerance to cancer therapies and poorer overall survival. Future research in this field should include more practical and precise definitions of the CC syndrome, making best use of available data, including cross-sectional imaging, circulating measurements and serial weight and BMI recordings. A better understanding of the origin, clinical significance and targetable vulnerabilities associated with $\mathrm{CC}$ in thoracic malignancy is required for development of specific treatments. This will require integration of both tumour (e.g. volumetric staging, genomics)97 and host biology and the conduct of large scale randomised clinical trials. 


\section{KEY POINTS:}

- Cancer cachexia (CC) remains a largely understudied and untreated condition

- $\mathrm{CC}$ results in reduced tolerance to treatment and poorer overall survival

- Future steps in the identification of $\mathrm{CC}$ should combine routinely available clinical tests such as serial weight and BMI measurements, inflammatory indices and crosssectional imaging

\section{ACKNOWLEDGMENTS:}

Acknowledgements: none.

Financial support and sponsorship: none.

Conflicts of interest: none. 


\section{REFERENCES AND RECOMMENDED READING}

1. Fearon K, Strasser F, Anker SD, et al. Definition and classification of cancer cachexia: an international consensus. Lancet Oncology 2011 May; 12(5): 489-95

2. Argiles JM, Busquets S, Stemmler B, et al. Cancer cachexia: understanding the molecular basis. Nature Reviews Cancer 2014; 14: 754-62

3. von Haehling S, Anker SD. Cachexia as a major underestimated and unmet medical need: facts and numbers. Journal of Cachexia, Sarcopenia and Muscle 2010; 1: 1-5

4. Muscaritoli M, Bossola M, Aversa Z, et al. Prevention and treatment of cancer cachexia: new insights into an old problem. European Journal of Cancer 2006; 42: $31-41$

5. Fortunati N, Manti R, Birocco N, et al. Pro-inflammatory cytokines and oxidative stress/antioxidant parameters characterize the bio-humoral profile of early cachexia in lung cancer patients. Oncology Reports 2007; 18: 1521-7

6. Vagnildhaug OM, Balstad TR, Almberg SS, et al. A cross-sectional study examining the prevalence of cachexia and areas of unmet need in patients with cancer. Supportive Care in Cancer 2018; 26(6): 1871-80

a. *This paper determined the prevalence of cancer cachexia across a range of different settings and disease sub-types

7. Dewys WD, Begg C, Lavin PT, et al. Prognostic effect of weight loss prior to chemotherapy 386 in cancer patients. Eastern Cooperative Oncology Group. American Journal of Medicine 1980; 69: 491-7

8. Buccheri G, Ferrigno D. Lung tumor markers of cytokeratin origin: an overview. Lung Cancer 2001; 34 Suppl 2: S65-9 
9. Ross PJ, Ashley S, Norton A, et al. Do patients with weight loss have a worse outcome when undergoing chemotherapy for lung cancers? British Journal of Cancer 2004; 90(10): 1905-11

10. Sanders KJC, Hendriks LE, Troost EGC, et al. Early Weight Loss during Chemoradiotherapy Has a Detrimental Impact on Outcome in NSCLC. Journal of Thoracic Oncology 2016; 11(6): 873-9

11. Martin L, Senesse P, Gioulbasanis I, et al. Diagnostic criteria for the classification of cancer-associated weight loss. Journal of Clinical Oncology 2015; 33; 90-9

12. Mytelka DS, Li L, Benoit K. Post - diagnosis weight loss as a prognostic factor in non - small cell lung cancer. Journal of Cachexia, Sarcopenia and Muscle 2018; 9(1): 86-92

a. *This paper assessed weight loss in a large cohort $(n=12,101)$ of patients with non-small cell lung cancer

13. Patel JD, Pereira JR, Chen J, et al. Relationship between efficacy outcomes and weight gain during treatment of advanced, non-squamous, non-small-cell lung cancer patients. Annals of Oncology 2016; 27(8): 1612-9

14. Masel EK, Berghoff AS, Fureder LM, et al. Decreased body mass index is associated with impaired survival in lung cancer patients with brain metastases: A retrospective analysis of 624 patients. European Journal of Cancer Care 2017; 26(6): 62017

15. Herndon JE, Green MR, Chahinian AP, et al. Factors predictive of survival among 337 patients with mesothelioma treated between 1984 and 1994 by the Cancer and Leukemia Group B. Chest 1998; 113(3): 723-31 
16. Edwards JG, Abrams KR, Leverment JN, et al. Prognostic factors for malignant mesothelioma in 142 patients: validation of CALGB and EORTC prognostic scoring systems. Thorax 2000; 55(9): 731-5

17. Meniawy TM, Creaney J, Lake RA, et al. Existing models, but not neutrophil-tolymphocyte ratio, are prognostic in malignant mesothelioma. British Journal of Cancer 2013; 109(7): 1813-20

18. Rusch VW, Piantadosi S, Holmes EC. The role of extrapleural pneumonectomy in malignant pleural mesothelioma. A Lung Cancer Study Group trial. Journal of Thoracic Cardiovascular Surgery 1991; 102(1): 1-9

19. Curran D, Sahmoud T, Therasse P, et al. Prognostic factors in patients with pleural mesothelioma: the European Organization for Research and Treatment of Cancer experience. Journal of Clinical Oncology 1998; 16(1): 145-52

20. Bottomley A, Aaronson NK; European Organisation for Research and Treatment of Cancer. International perspective on health-related quality-of-life research in cancer clinical trials: the European Organisation for Research and Treatment of Cancer experience Journal of Clinical Oncology 2007; 25(32): 5082-6

21. Kidd AC, McGettrick M, Tsim S, et al. Survival prediction in mesothelioma using a scalable Lasso regression model: instructions for use and initial performance using clinical predictors. BMJ Open Respiratory Research 2018; 30; 5(1): e000240

22. Davis MP, Dreicer R, Walsh D, et al. Appetite and cancer-associated anorexia: a review. Journal of Clinical Oncology 2004; 22(8): 1510-7

23. Krasnow SM, Marks DL. Neuropeptides in the Pathophysiology and Treatment of Cachexia. Current Opinion in Supportive and Palliative Care 2010; 4(4): 266-71 
24. Karapanagiotou EM, Polyzos A, Dilana KD, et al. Increased serum levels of ghrelin at diagnosis mediate body weight loss in non-small cell lung cancer (NSCLC) patients. Lung Cancer 2009; 66(3): 393-8

25. Blauwhoff-Buskermolen S, Langius JA, Heijboer AC, et al. Plasma ghrelin levels are associated with anorexia but not cachexia in patients with NSCLC. Frontiers in Physiology 2017; 8: 119

26. Khatib MN, Gaidhane A, Gaidhane S, et al. Ghrelin as a Promising Therapeutic Option for Cancer Cachexia. Cellular Physiology and Biochemistry 2018; 48(5): $2172-88$

27. Asakawa A, Fujimiya M, Niijima A, et al. Parathyroid hormone-related protein has an anorexigenic activity via activation of hypothalamic urocortins 2 and 3 . Psychoneuroendocrinology 2010; 35(8): 1178-86

28. Muers MF, Round CE. Palliation of symptoms in non-small cell lung cancer: a study by the Yorkshire Regional Cancer Organisation Thoracic Group. Thorax 1993; 48: $339-43$

29. Lindsey AM, Piper BF. Anorexia and weight loss: indicators of cachexia in small cell lung cancer. Nutrition and Cancer 1985; 7(1-2): 65-76

30. Kao SC, Vardy J, Harvie R, et al. Health-related quality of life and inflammatory markers in malignant pleural mesothelioma. Supportive Care in Cancer 2013; 21(3): $697-705$

31. Moley JF, Aamodt R, Rumble W, et al. Body cell mass in cancer-bearing and anorexic patients. JPEN Journal of Parenteral \& Enteral Nutrition 1987;11: 219-22

32. Fredrix EW, Soeters PB, Wouters EF, et al. Effect of different tumor types on resting energy expenditure. Cancer Research 1991; 51(22): 6138-41 
33. Jatoi A, Daly BD, Hughes VA, et al. Do patients with nonmetastatic non-small cell lung cancer demonstrate altered resting energy expenditure? Annals of Thoracic Surgery 2001; 72(2): 348-51

34. Sengelov H, Hansen OP, Simonsen L, et al. Inter-relationships between single carbon units' metabolism and resting energy expenditure in weight-losing patients with small cell lung cancer. Effects of methionine supply and chemotherapy. European Journal of Cancer 1994; 30A(11): 1616-20

35. Jebb SA, Osborne RJ, Dixon AK, et al. Measurements of resting energy expenditure and body composition before and after treatment of small cell lung cancer. Annals of Oncology 1994; 5(10): 915-9

36. Staal-van den Brekel AJ, Schols AM, Dentener MA, et al. Metabolism in patients with small cell lung carcinoma compared with patients with non-small cell lung carcinoma and healthy controls. Thorax 1997; 52(4): 338-41

37. Staal-van Den Brekel AJ, Dentener MA, Schols AMWJ, et al. Increased resting energy expenditure and weight loss are related to a systemic inflammatory response in lung cancer patients. Journal of Clinical Oncology 1995; 13(10): 2600-5

38. Derman BA, Macklis JN, Azeem MS, et al. Relationships between longitudinal neutrophil to lymphocyte ratios, body weight changes, and overall survival in patients with non-small cell lung cancer. BMC Cancer 2017; 17(1): 141

39. Kurishima K, Watanabe $\mathrm{H}$, Ishikawa $\mathrm{H}$, et al. Modified glasgow prognostic score in patients with small-cell lung cancer. Molecular and Clinical Oncology 2017; 7(1): $121-4$

40. Tsukioka T, Nishiyama N, Izumi N, et al. Sarcopenia is a novel poor prognostic factor in male patients with pathological Stage I non-small cell lung cancer. Japanese Journal of Clinical Oncology 2017; 47(4): 363-8 
a. *This paper found that sarcopenia was a predictor of poor prognosis in male patients with Stage I non-small cell lung cancer

41. Jafri SH, Shi R, Mills G. Advance lung cancer inflammation index (ALI) at diagnosis is a prognostic marker in patients with metastatic non-small cell lung cancer (NSCLC): a retrospective review. BMC Cancer 2013; 13: 158

42. Go SI, Park MJ, Song HN, et al. Sarcopenia and inflammation are independent predictors of survival in male patients newly diagnosed with small cell lung cancer. Supportive Care in Cancer 2016; 24(5): 2075-84

43. Kao SC, Pavlakis N, Harvie R, et al. High blood neutrophil-to-lymphocyte ratio is an indicator of poor prognosis in malignant mesothelioma patients undergoing systemic therapy. Clinical Cancer Research 2010; 16: 5805-13

44. Cihan YB, Ozturk A, Mutlu H. Relationship between prognosis and neutrophil: lymphocyte and platelet:lymphocyte ratios in patients with malignant pleural mesotheliomas. Asian Pacific Journal of Cancer Prevention 2014; 15: 2061-7

45. Nagata M, Ito H, Matsuzaki T, et al. Body mass index, C-reactive protein and survival in smokers undergoing lobectomy for lung cancer. European Journal of CardioThoracic Surgery 2017; 51(6): 1164-70

46. Ghanim B, Hoda MA, Winter MP, et al. Pretreatment serum C-reactive protein levels predict benefit from multimodality treatment including radical surgery in malignant pleural mesothelioma: a retrospective multicenter analysis. Annals of Surgery 2012; 256: $357-62$

47. Srdic D, Plestina S, Sverko-Peternac A, et al. Cancer cachexia, sarcopenia and biochemical markers in patients with advanced non-small cell lung cancerchemotherapy toxicity and prognostic value. Supportive Care in Cancer 2016; 24(11): $4495-502$ 
48. Yao ZH, Tian GY, Yang SX, et al. Serum albumin as a significant prognostic factor in patients with malignant pleural mesothelioma. Tumor Biology 2014; 35: 6839-45

49. Simmons CP, Koinis F, Fallon MT, et al. Prognosis in advanced lung cancer - A prospective study examining key clinicopathological factors. Lung Cancer 2015; 88(3): 304-9

50. Jeffery E, Lee YCG, Newton RU, et al. Body composition and nutritional status in malignant pleural mesothelioma: implications for activity levels and quality of life. European Journal of Clinical Nutrition 2019; doi: 10.1038/s41430-019-0418-9 [Epub ahead of print]

a. *This paper reported that patients with malignant pleural mesothelioma has high rates of pre-sarcopenia and malnutrition

51. Prado CMM, Baracos VE, McCargar LJ, et al. Body composition as an independent determinant of 5-fluorouracil-based chemotherapy toxicity. Clinical Cancer Research $2007 ; 13: 3264-8$

52. Martin L, Birdsell L, MacDonald N, et al. Cancer cachexia in the age of obesity: skeletal muscle depletion is a powerful prognostic factor, independent of body mass index. Journal of Clinical Oncology 2013; 31: 1539-47

53. Morley JE, Anker SD, von Haehling S. Prevalence, incidence, and clinical impact of sarcopenia: facts, numbers, and epidemiology-update 2014. Journal of Cachexia, Sarcopenia and Muscle 2014; 5(4): 253-9

54. Martínez-Hernández PL, Hernanz-Macías Á, Gómez-Candela C, et al. Serum interleukin-15 levels in cancer patients with cachexia. Oncology Reports 2012; 28(4): $1443-52$ 
55. Bovio G, Bettaglio R, Bonetti G, et al. Evaluation of nutritional status and dietary intake in patients with advanced cancer on palliative care. Minerva Gastroenterologica e Dietologica 2008; 54(3): 243-50

56. Prado CM, Lieffers JR, McCargar LJ, et al. Prevalence and clinical implications of sarcopenic obesity in patients with solid tumours of the respiratory and gastrointestinal tracts: a population-based study. Lancet Oncology 2008; 9(7): 629-35

57. Hansell DT, Davies JW, Shenkin A, et al. The oxidation of body fuel stores in cancer patients. Annals of Surgery 1986; 204(6): 637-42

58. Fredrix EW, Soeters PB, von Meyenfeldt MF, et al. Measurement of resting energy expenditure in a clinical setting. Clinical Nutrition 1990; 9(6): 299-304

59. Tozer RG, Tai P, Falconer W, et al. Cysteine-rich protein reverses weight loss in lung cancer patients receiving chemotherapy or radiotherapy. Antioxidants and Redox Signaling 2008; 10(2): 395-402

60. Crown AL, Cottle K, Lightman SL, et al. What is the role of the insulin-like growth factor system in the pathophysiology of cancer cachexia, and how is it regulated? Clinical Endocrinology 2002; 56(6): 723-33

61. Beijer S, Hupperets PS, van den Borne BE, et al. Effect of adenosine 5'-triphosphate infusions on the nutritional status and survival of preterminal cancer patients. Anticancer Drugs 2009; 20(7): 625-33

62. Suzuki Y, Okamoto T, Fujishita T, et al. Clinical implications of sarcopenia in patients undergoing complete resection for early non-small cell lung cancer. Lung Cancer 2016: 16; 92-7

63. Hervochon R, Bobbio A, Guinet C, et al. Body Mass Index and Total Psoas Area Affect Outcomes in Patients Undergoing Pneumonectomy for Cancer. Annals of Thoracic Surgery 2017; 103(1): 287-95 
a. *Another paper that reported sarcopenia prevalence in early stage non-small cell lung cancer

64. Shoji F, Matsubara T, Kozuma Y, et al. Relationship Between Preoperative Sarcopenia Status and Immuno-nutritional Parameters in Patients with Early-stage Non-small Cell Lung Cancer. Anticancer Research 2017; 37(12): 6997-7003

65. Kim EY, Lee HY, Kim KW, et al. Preoperative Computed Tomography-Determined Sarcopenia and Postoperative Outcome After Surgery for Non-Small Cell Lung Cancer. Scandinavian Journal of Surgery 2018; 107(3): 244-51

66. Nakamura R, Inage Y, Tobita R, et al. Sarcopenia in Resected NSCLC: Effect on Postoperative Outcomes. Journal of Thoracic Oncology 2018; 13(7): 895-903

67. Deng HY, Hou L, Zha P, et al. Sarcopenia is an independent unfavorable prognostic factor of non-small cell lung cancer after surgical resection: A comprehensive systematic review and meta-analysis. European Journal of Surgical Oncology 2019; 45(5): 728-35

68. Kim EY, Kim YS, Park I, et al. Prognostic Significance of CT-Determined Sarcopenia in Patients with Small-Cell Lung Cancer. Journal of Thoracic Oncology $2015 ; 10(12): 1795-9$

69. Kazemi-Bajestani SM, Mazurak VC, Baracos V. Computed tomography-defined muscle and fat wasting are associated with cancer clinical outcomes. Seminars in Cell and Developmental Biology 2016; 54: 2-10

70. Nattenmüller J, Wochner R, Muley T, et al. Prognostic Impact of CT-Quantified Muscle and Fat Distribution before and after First-Line-Chemotherapy in Lung Cancer Patients. PLoS One 2017; 12(1): e0169136 
71. Kim EY, Lee HY, Kim YS, et al. Prognostic significance of cachexia score assessed by CT in male patients with small cell lung cancer. European Journal of Cancer Care (Engl) 2018; 27(1): e12695

a. *This paper determines the prognostic significance of CT-determined cachexia scores male patients with small cell lung cancer

72. Matsuo Y, Mitsuyoshi T, Shintani T, et al. Impact of low skeletal muscle mass on non-lung cancer mortality after stereotactic body radiotherapy for patients with stage I non-small cell lung cancer. Journal of Geriatric Oncology 2018; 9(6): 589-93

73. Kimura M, Naito T, Kenmotsu H, et al. Prognostic impact of cancer cachexia in patients with advanced non-small cell lung cancer. Supportive Care in Cancer 2015; 23(6): 1699-708

74. Cehreli R, Yavuzsen $\mathrm{T}$, Ates $\mathrm{H}$, et al. Can inflammatory and nutritional serum markers predict chemotherapy outcomes and survival in advanced stage non-small cell lung cancer patients? BioMed Research International 2019: 1648072

75. Sjøblom B, Benth JŠ, Grønberg BH, et al. Drug Dose Per Kilogram Lean Body Mass Predicts Hematologic Toxicity From Carboplatin-Doublet Chemotherapy in Advanced Non-Small-Cell Lung Cancer. Clinical Lung Cancer 2017; 18(2): e129-e36

a. *This paper highlighted that dose per kilogram of lean body mass was an independent predictor of haematological toxicity in patients with non-small cell lung cancer

76. Arrieta O, De la Torre-Vallejo M, López-Macías D, et al. Nutritional Status, Body Surface, and Low Lean Body Mass/Body Mass Index Are Related to Dose Reduction and Severe Gastrointestinal Toxicity Induced by Afatinib in Patients With Non-Small Cell Lung Cancer. Oncologist 2015; 20(8): 967-74 
77. Bowden JCS, Williams LJ, Simms A, et al.. Prediction of 90 Day and Overall Survival after Chemoradiotherapy for Lung Cancer: Role of Performance Status and Body Composition. Clinical Oncology 2017; 29(9): 576-84

78. Kakinuma K, Tsuruoka H, Morikawa K, et al. Differences in skeletal muscle loss caused by cytotoxic chemotherapy and molecular targeted therapy in patients with advanced non-small cell lung cancer. Thoracic Cancer 2018; 9(1): 99-104

a. This paper reported that skeletal muscle loss was lower in non-small cell lung cancer patients receiving targeted therapy than in patients receiving cytotoxic chemotherapy

79. Cortellini A, Verna L, Porzio G, et al. Predictive value of skeletal muscle mass for immunotherapy with nivolumab in non-small cell lung cancer patients: A "hypothesis-generator" preliminary report. Thoracic Cancer 2019; 10(2): 347-51

80. Takamori S, Toyokawa G, Okamoto T, et al. Clinical Impact and Risk Factors for Skeletal Muscle Loss After Complete Resection of Early Non-small Cell Lung Cancer. Annals of Surgical Oncology 2018; 25(5): 1229-36

81. Kimura M, Naito T, Kenmotsu H, et al. Prognostic impact of cancer cachexia in patients with advanced non-small cell lung cancer. Supportive Care in Cancer 2015; 23(6): 1699-708

82. Kim EY, Kim YS, Park I, et al. Evaluation of sarcopenia in small-cell lung cancer patients by routine chest CT. Supportive Care in Cancer 2016; 24(11): 4721-6

83. Furtner J, Berghoff AS, Albtoush OM, et al. Survival prediction using temporal muscle thickness measurements on cranial magnetic resonance images in patients with newly diagnosed brain metastases. European Radiology 2017; 27(8): 3167-73

84. Lee JW, Lee HS, Na JO, et al. Effect of adipose tissue volume on prognosis in patients with non-small cell lung cancer. Clinical Imaging 2018; 50: 308-13 
85. Bauer JD, Capra S. Nutrition intervention improves outcomes in patients with cancer cachexia receiving chemotherapy - a pilot study. Supportive Care in Cancer 2005; 13(4): $270-4$

86. Tanaka N, Takeda K, Kawasaki Y, et al. Early intensive nutrition intervention with dietary counseling and oral nutrition supplement prevents weight loss in patients with advanced lung cancer receiving chemotherapy: A clinical prospective study. Yonago Acta Medica 2018; 61(4): 204-12

87. Moertel CG, Schutt AJ, Reitemeier RJ, et al. Corticosteroid therapy of preterminal gastrointestinal cancer. Cancer 1974; 33: 1607-9

88. Loprinzi CL, Kugler JW, Sloan JA, et al. Randomized comparison of megestrol acetate versus dexamethasone versus fluoxymesterone for the treatment of cancer anorexia/cachexia. Journal of Clinical Oncology 1999; 17: 3299-3306.

89. Temel JS, Abernethy AP, Currow DC, et al. Anamorelin in patients with non-smallcell lung cancer and cachexia (ROMANA 1 and ROMANA 2): results from two randomised, double-blind, phase 3 trials. Lancet Oncology 2016; 17(4): 519-31

90. Currow D, Temel JS, Abernethy A, et al. ROMANA 3: a phase 3 safety extension study of anamorelin in advanced non-small-cell lung cancer (NSCLC) patients with cachexia. Annals of Oncology 2017; 28(8): 1949-56

a. This paper explores the use of Anamorelin in patients with advanced nonsmall-cell lung cancer and cancer cachexia

91. Stewart Coats AJ, Ho GF, Prabhash K, et al. Espindolol for the treatment and prevention of cachexia in patients with stage III/IV non-small cell lung cancer or colorectal cancer: a randomized, double-blind, placebo-controlled, international multicentre phase II study (the ACT-ONE trial). Journal of Cachexia, Sarcopenia and Muscle 2016; 7(3): 355-65 
92. Solomon ZJ, Mirabal JR, Mazur DJ, et al. Selective Androgen Receptor Modulators: Current Knowledge and Clinical Applications. Sexual Medicine Reviews 2019; 7(1): 84-94

93. Crawford J, Prado CM, Johnston MA, et al. Study Design and Rationale for the Phase 3 Clinical Development Program of Enobosarm, a Selective Androgen Receptor Modulator, for the Prevention and Treatment of Muscle Wasting in Cancer Patients (POWER Trials). Current Oncology Reports 2016; 18(6): 37

94. GTx reports results for enobosarm POWER trials for the prevention and treatment of muscle wasting in patients with non-small cell lung cancer. 2013 [cited August 12, 2019] Available from: http://www.businesswire.com/news/home/20130819005378/en/GTx-Reports-ResultsEnobosarm-POWER-Trials-Prevention

95. Solheim TS, Laird BJA, Balstad TR, et al. A randomized phase II feasibility trial of a multimodal intervention for the management of cachexia in lung and pancreatic cancer. Journal of Cachexia, Sarcopenia and Muscle 2017; 8: 778-88

a. **This paper determined the feasibility of a multi-modal cancer cachexia intervention

96. Naito T, Mitsunaga S, Miura S, et al. Feasibility of early multimodal interventions for elderly patients with advanced pancreatic and non-small-cell lung cancer. Journal of Cachexia, Sarcopenia and Muscle 2019; 10 (1): 73-83

97. Antoun S, Morel H, Souquet PJ, et al. Staging of nutrition disorders in non-small-cell lung cancer patients: utility of skeletal muscle mass assessment. Journal of Cachexia, Sarcopenia and Muscle 2019; 10(4): 782-93 